\title{
STRUCTURE CHARACTERIZATION OF ZnSe/GaMnAs QUANTUM WELL ON GaAs SUBSTRATE
}

\author{
G. D. Lian*, E. C. Dickey*, S. H. Chun**, N. Samarth**
}

*Department of Materials Science and Engineering, The Pennsylvania State University, University Park, PA 16802.

** Department of Physics, The Pennsylvania State University, University Park, PA 16802

There is substantial interest in exploiting to use both the charge and spin of electron in semiconductor devices ${ }^{1)}$. In order to study the spin transfer across herterostructures, $\mathrm{Ga}_{1-\mathrm{x}} \mathrm{Mn}_{\mathrm{x}} \mathrm{As}$ /ZnSe quantum well (QW) has been growth on semi-insulating GaAs substrate. Previous study indicated that the structure defects play a critical role in the ferromagnetism of the materials ${ }^{2)}$. Also the concentration of manganese in GaAs is an important factor for the magnetic properties of the structure $^{3)}$. Here, we report detail structure characterization and composition analysis on $\mathrm{Ga}_{1 \text { - }}$ ${ }_{\mathrm{x}} \mathrm{Mn}_{\mathrm{x}} \mathrm{As} / \mathrm{ZnSe}$ quantum well on GaAs substrate.

The sample was grown by molecular beam epitaxy on semi-insulating (100) GaAs substrate. ${ }^{4)}$ The epitaxial layers include $350 \mathrm{~nm} \mathrm{ZnSe}$ buffer layer, 50nm $\mathrm{Ga}_{0.95} \mathrm{Mn}_{0.05} \mathrm{As}$, and $350 \mathrm{~nm} \mathrm{ZnSe}$. Structural and chemical characterization was carried in a JEOL 2010F field emission TEM/STEM, equipped with an annular dark field detector, post-column EELS image filter (Gatan GIF200) and Oxford energy dipersive x-ray (EDX) detector.

Figure 1 is a bright field image from cross-section view showing the overall structure, which reveals three distinct layers over GaAs substrate. Figure 2 shows HREM images of three interfaces taken from [001] zone axis, which had been demonstrated the most chemical sensitive beam direction for HREM imaging ${ }^{5)}$. Atomically abrupt interfaces were clearly shown in the images. Slightly fluctuate in lattice contrast at GaMnAs/ZnSe was due to the fact that the thickness variation changing the local defocus. No defect has been found at the interfaces and thin films layers from HREM images. Figure 3 shows EELS spectra collected from ZnSe and GaMnAs layers. The inset in figure 3 exhibits the $\mathrm{Mn} \mathrm{L}_{2,3}$ edge, a part of spectrum (also containing $\mathrm{Ga} \mathrm{L}_{2,3}$ edge) acquired from GaMnAs layer. From EELS spectra, the atomic ratio of Mn:Ga:As was quantified to be 0.07:0.93:1 in GaMnAs layer by using Hartree-Slater model. Figure 4 is the EELS spectrum profile across GaMnAs layer, as indicated in the inset annual dark field (ADF) image. The abrupt composition changes at both interfaces imply that no inter-diffusion between the ZnSe and GaMnAs layers.

References

1. H. Ohno, Science, 281 (1998) 951

2. S. J. Potashnik, K. C. Ku, S. H. Chun, J. J. Berry, N. Samarth, and P. Schiffer, Appl. Phys. Lett.79 (2001) 1495.

3. H. Ohno, F. Matsukura, Solid State Comm. 117 (2001) 179.

4. H. Chun, N. Samarth, G. D. Lian and E. C. Dickey to be submitted to Appl. Phys. Lett.

5. A. Ourmazd, D. W. Tayler, J. Cunningham, and C. W Tu, Phys. Rew. Lett. 62 (1989) 933. 


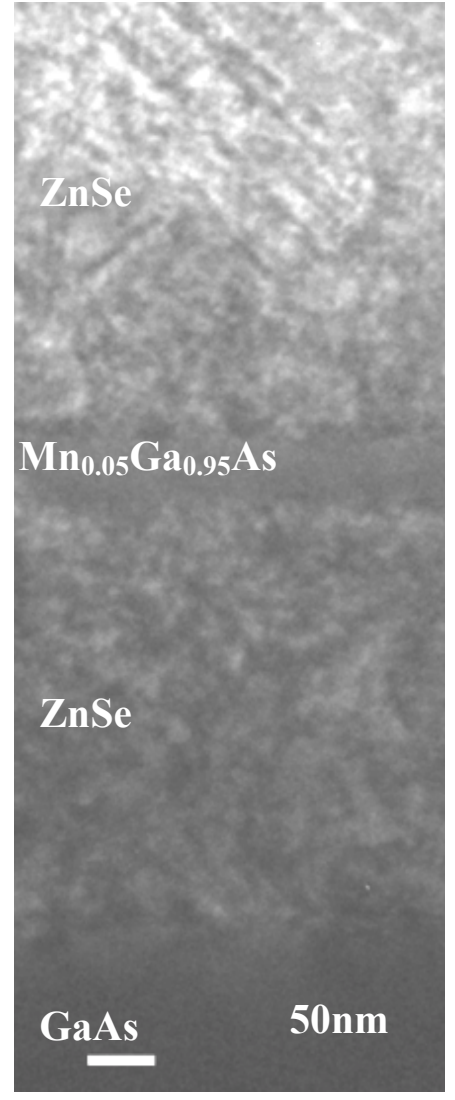

1)

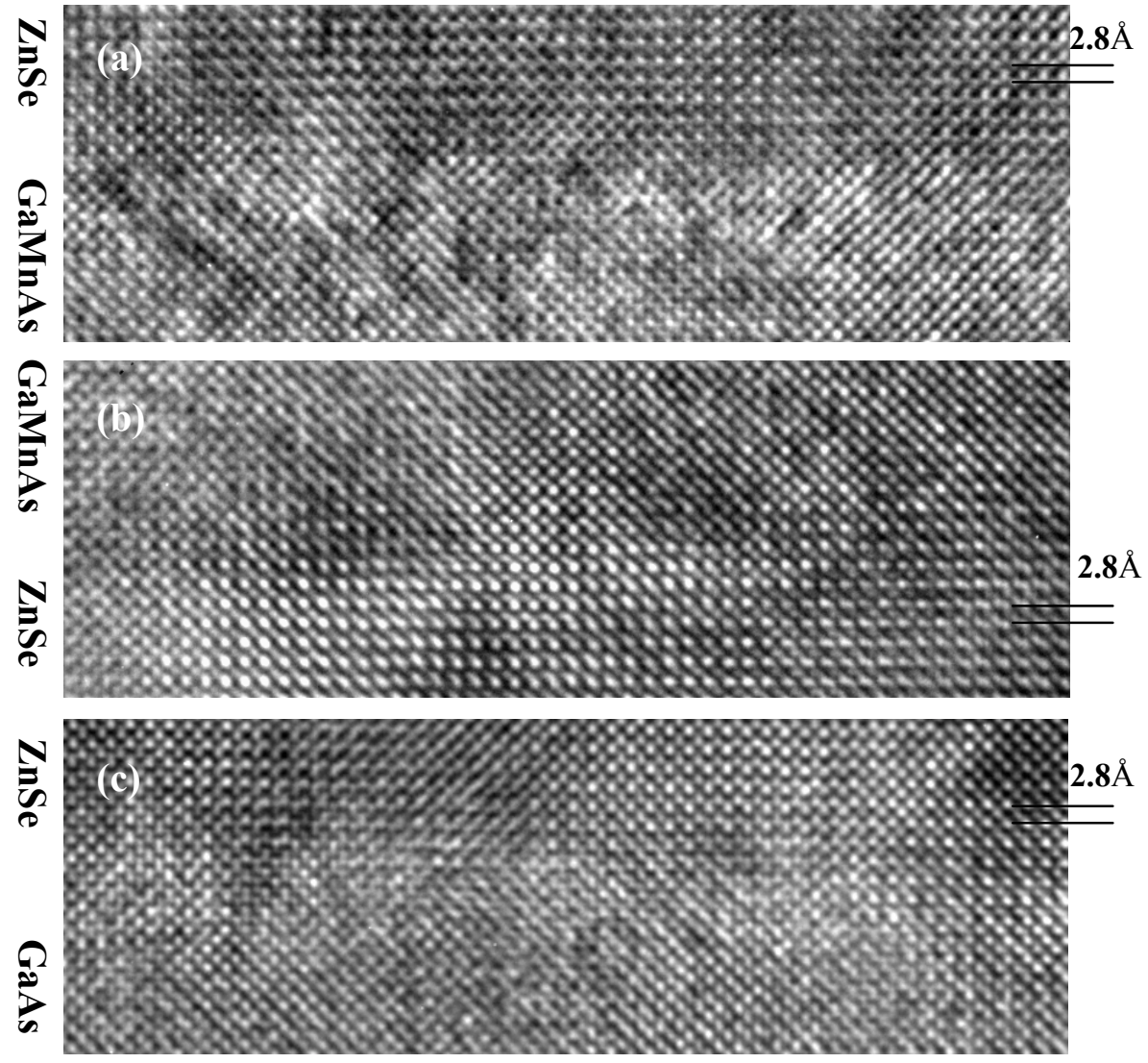

2)
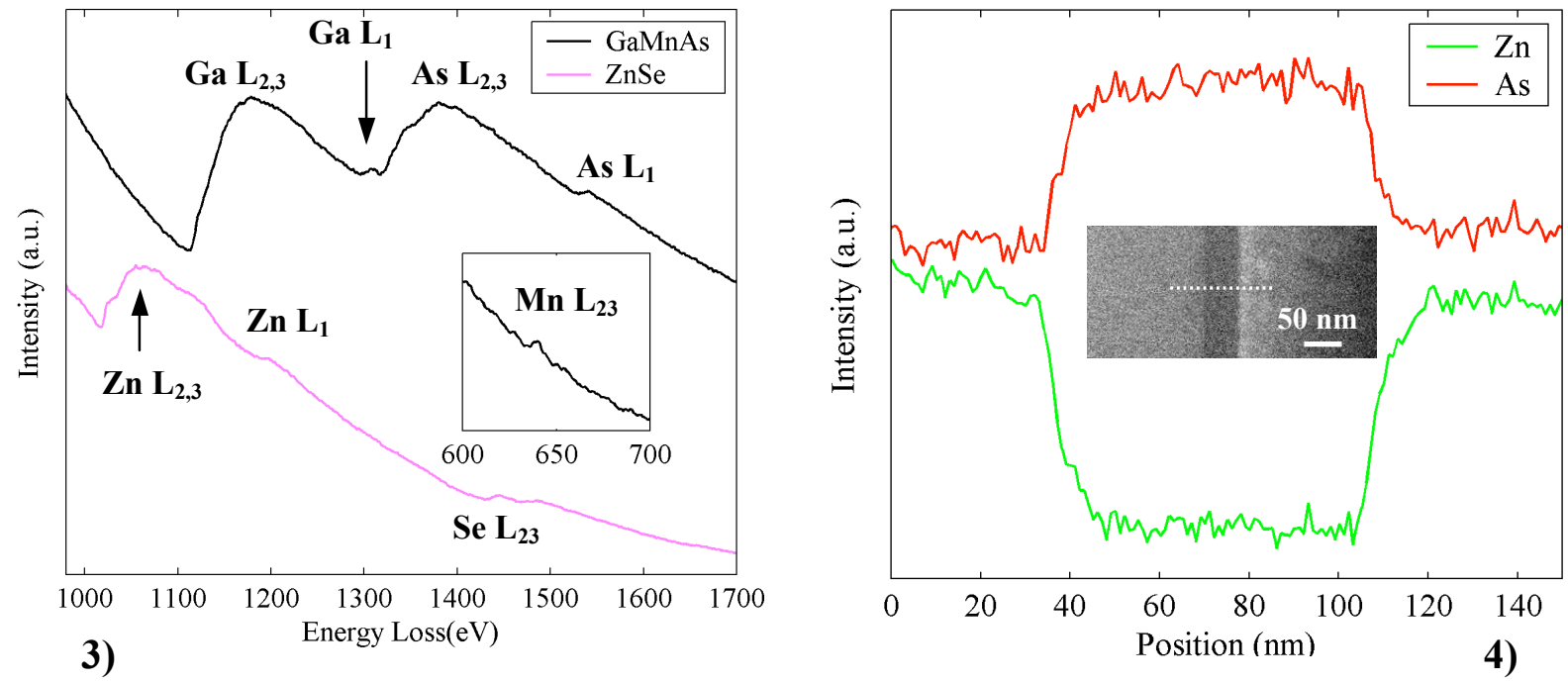

Fig 1. Bright field image from cross section sample.

Fig 2. HREM images of $\mathrm{ZnSe} / \mathrm{GaMnAs}$ (a), GaMnAs /ZnSe (b), and $\mathrm{ZnSe} / \mathrm{GaAs}$ (c) interfaces. Fig 3. EELS spectra collected from GaMnAs and ZnSe layers, inset shows Mn L2,3 edge. Fig 4. EELS spectrum profile across GaMnAs layer as indicated from inset ADF image. 